\title{
Conserved structural motif identified in peptides that bind to Geminivirus replication protein Rep
}

J. Trinidad Ascencio-Ibáñez ${ }^{1}$ and Benjamin G. Bobay ${ }^{2,3,4, *}$

${ }^{1}$ Department of Molecular and Structural Biochemistry, North Carolina State University, Raleigh,

NC 27695, USA.

${ }^{2}$ Department of Biochemistry, Duke University, Durham, NC 27710, USA.

${ }^{3}$ Department of Radiology, Duke University, Durham, NC 27710, USA.

${ }^{4}$ Duke University NMR Center, Duke University Medical Center, Durham, NC 27710, USA. 


\section{Supplemental Figures}

Supplemental Figure 1.

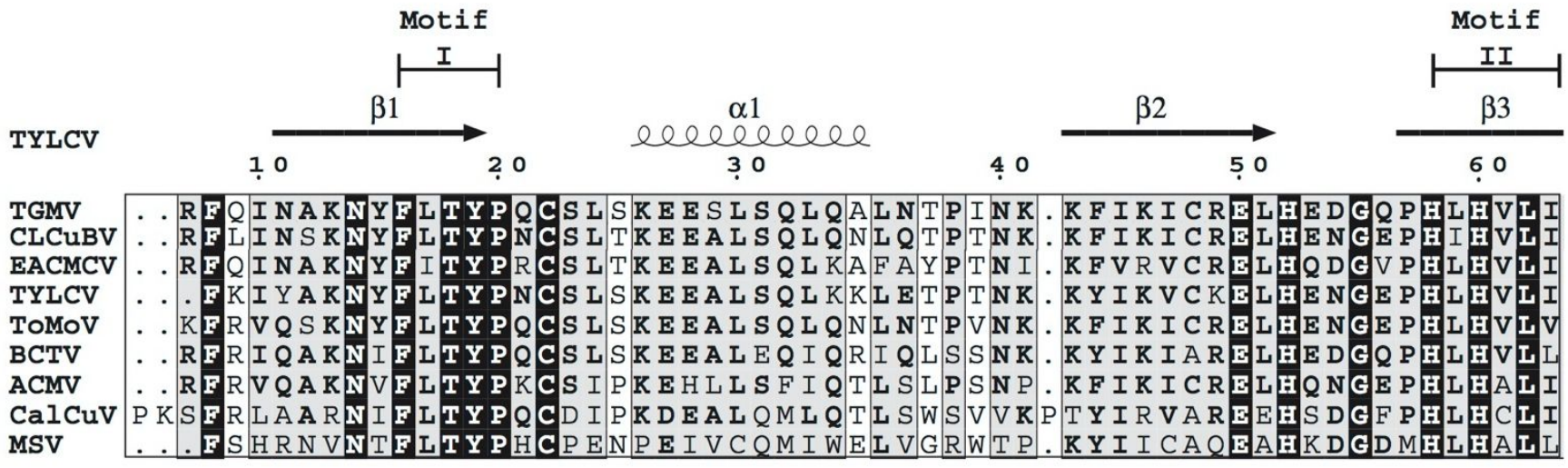

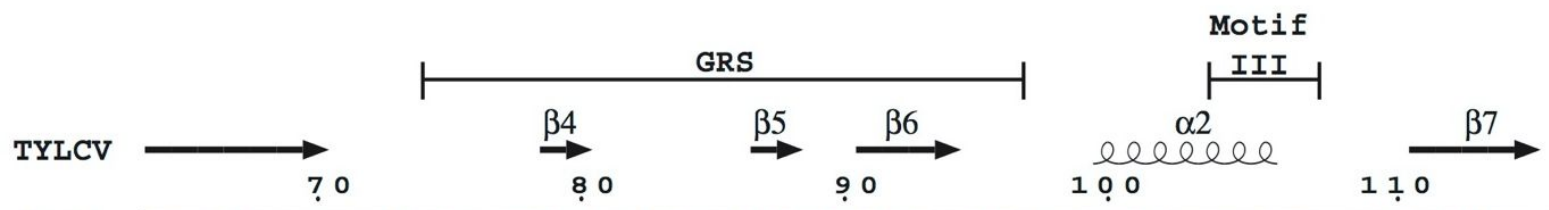

TGMV QF E GKY C C $Q$ NRFF DLVS PT R S AHFH P I QRARS S S DVKTY I DKD. G D TLVWGE F QV D GR

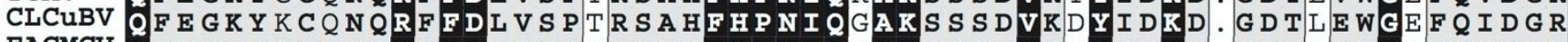

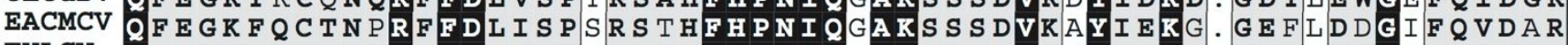
TYLCV QFEGKYQCKNQRFFDLVSPNRSAHFHPNIQAAKS T DVKTYVEKD. GNFIDFGVSQIDGR TOMOV QFEGKYQCTNNRF DLVSPTRSAHFHPNI QGAKS S DVK SYIDKD. GDTIEWGDFQIDGR BCTV QLEGKVQITNIRLFDLVSPTRSAHFHPNIQRAKS S DVKSYVDKD. GDTIEWGEFQIDGR

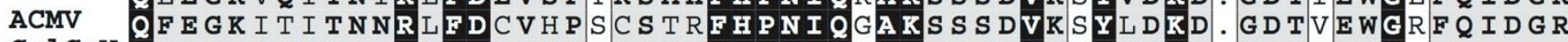
CalCuV QLSGKNIKDARFFDITHPRRSANFHPNIQAAKDTNAVKNYITKD. GDYCESG. MSV QTEKPVRITDSRFFDIEG. . . . FHPNIOSARSVNKVRDYILKE P LAVFERGTF I PRKS

Supplemental Figure 1. A sequence alignment of $\mathbf{N}$-term Rep proteins used in this study. TYLCV secondary structure as characterized in 1L5I PDB and conserved motifs are shown. 
Supplemental Figure 2.

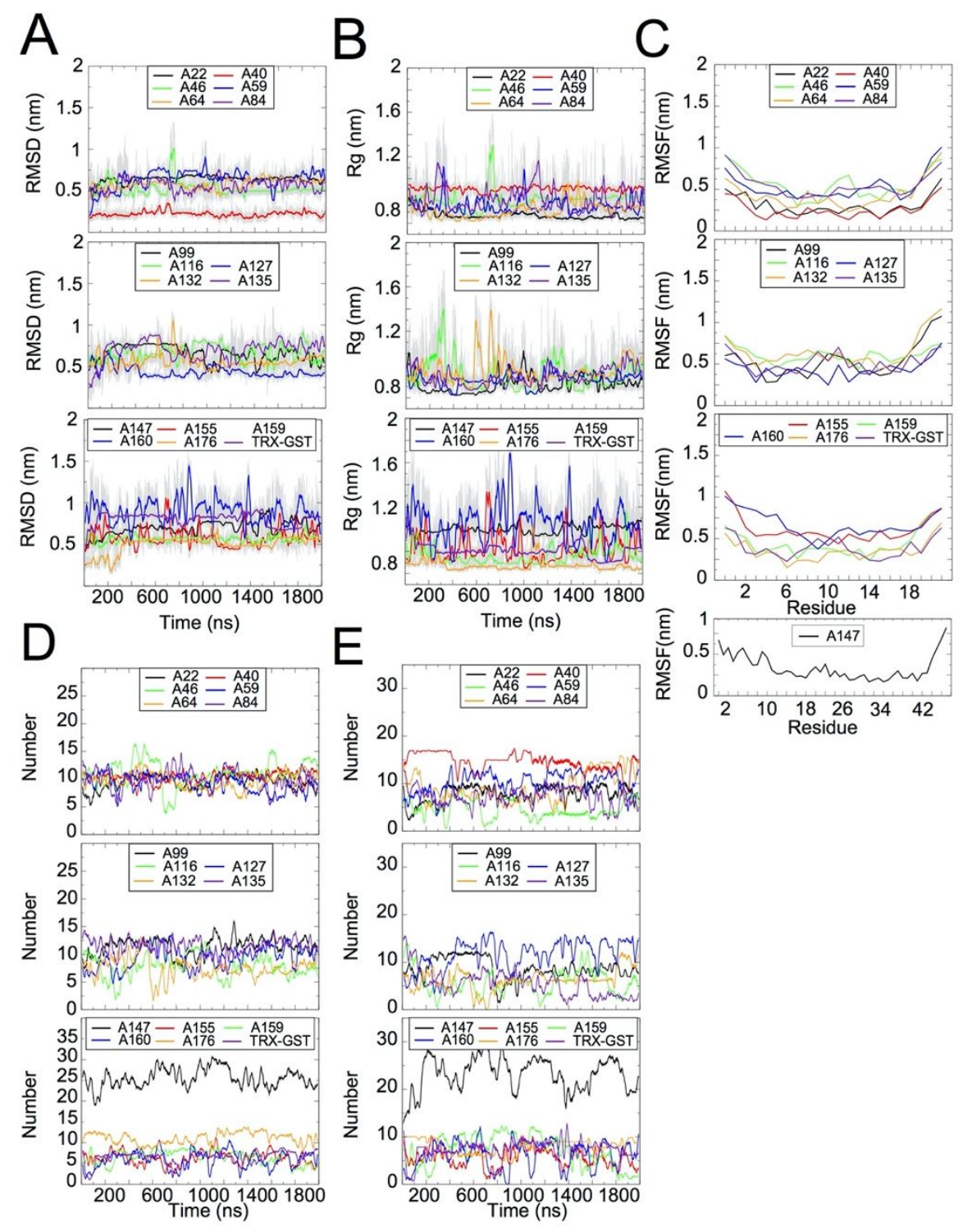

Supplemental Figure 2. Peptide analysis of the $2 \mu$ s peptide MD simulation. (A), C $\alpha$-RMSD over the length of the simulation. (B), $\operatorname{Rg}$ (radius of gyration over the length of the simulation). (C), root mean square fluctuations (RMSF) per residue, A147 removed from the rest as there is 47 residues compared to the $\sim 20$ residues for each other peptide. (D), number of hydrogen bonds in total per peptide. (E), number of residues in a secondary structure confirmation per peptide $(\alpha-$ Helix $+\beta$-Sheet $+\beta$-Bridge + Turn). The 18 peptides are divided up into 3 figures each within each panel: peptides A22 (black), A40 (red), A46 (green), A59 (blue), A64 (orange), and A84 (purple) (top); A99 (black), A116 (green), A127 (blue), A132 (orange), and A135 (purple) (middle); and A147 (black), A155 (red), A159 (green), A160 (blue), A176 (orange), and TRXGST (purple) (bottom). Raw data is shown in grey lines while colored lines are the running averages in panels A and B. 
Supplemental Figure 3.

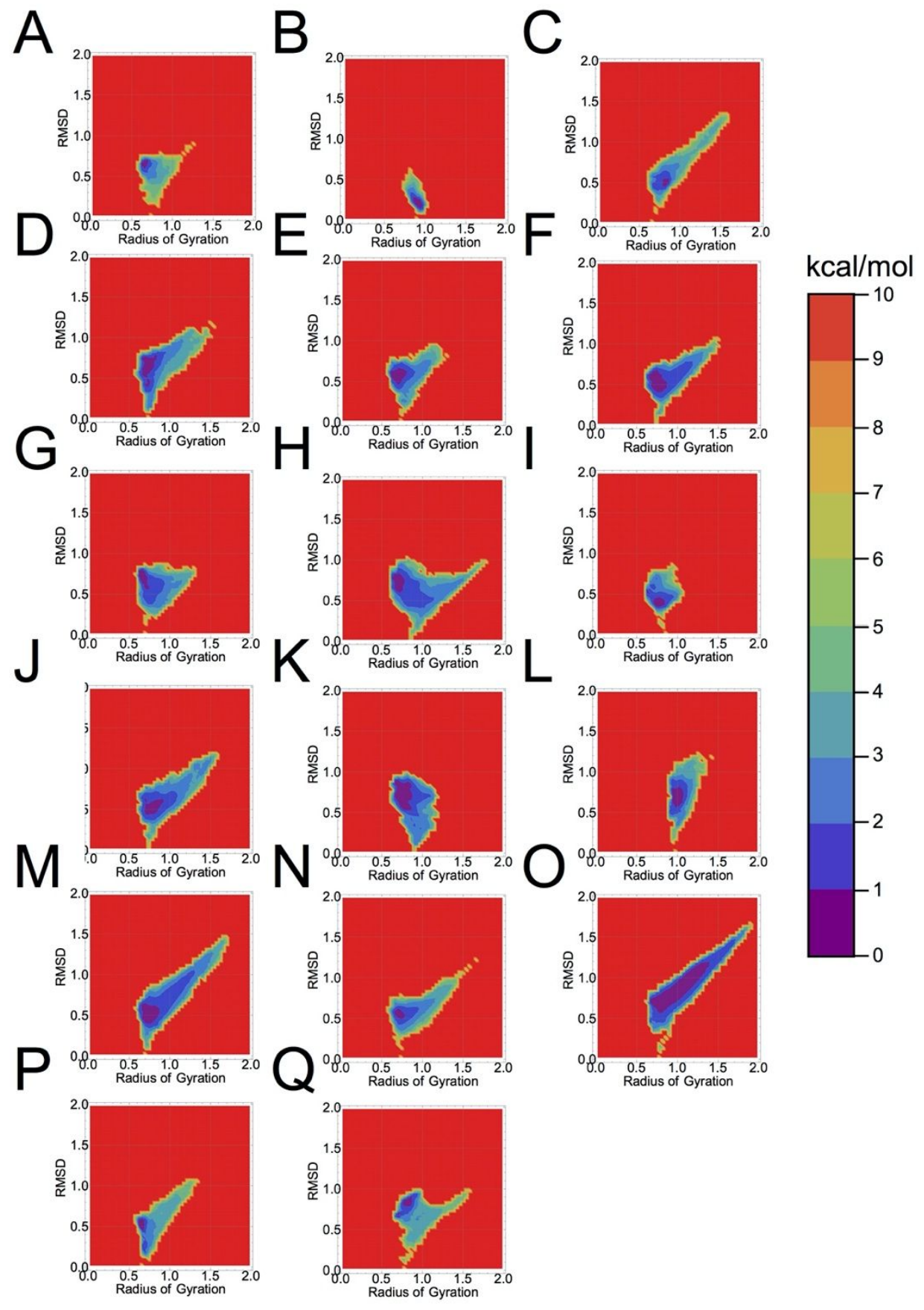

Supplemental Figure 3. Free energy landscape analysis of the $\mathbf{2} \boldsymbol{\mu}$ s peptide MD simulation. Peptides A22 (A), A40 (B), A46 (C), A59 (D), A64 (E), A84 (F), A99 (G), A116 (H), A127 (I), A132 (J), A135 (K), A147 (L), A155 (M), A159 (N), A160 (O), A176 (P), and TRX-GST (Q) FEL analysis. The FEL values are constructed as a function of projections of the MD trajectory onto their own $\mathrm{C} \alpha$-RMSD and $\mathrm{C} \alpha$-Rg values, respectively. The color bar represents the relative free-energy value in $\mathrm{kcal} \mathrm{mol}^{-1}$. 
Supplemental Figure 4.

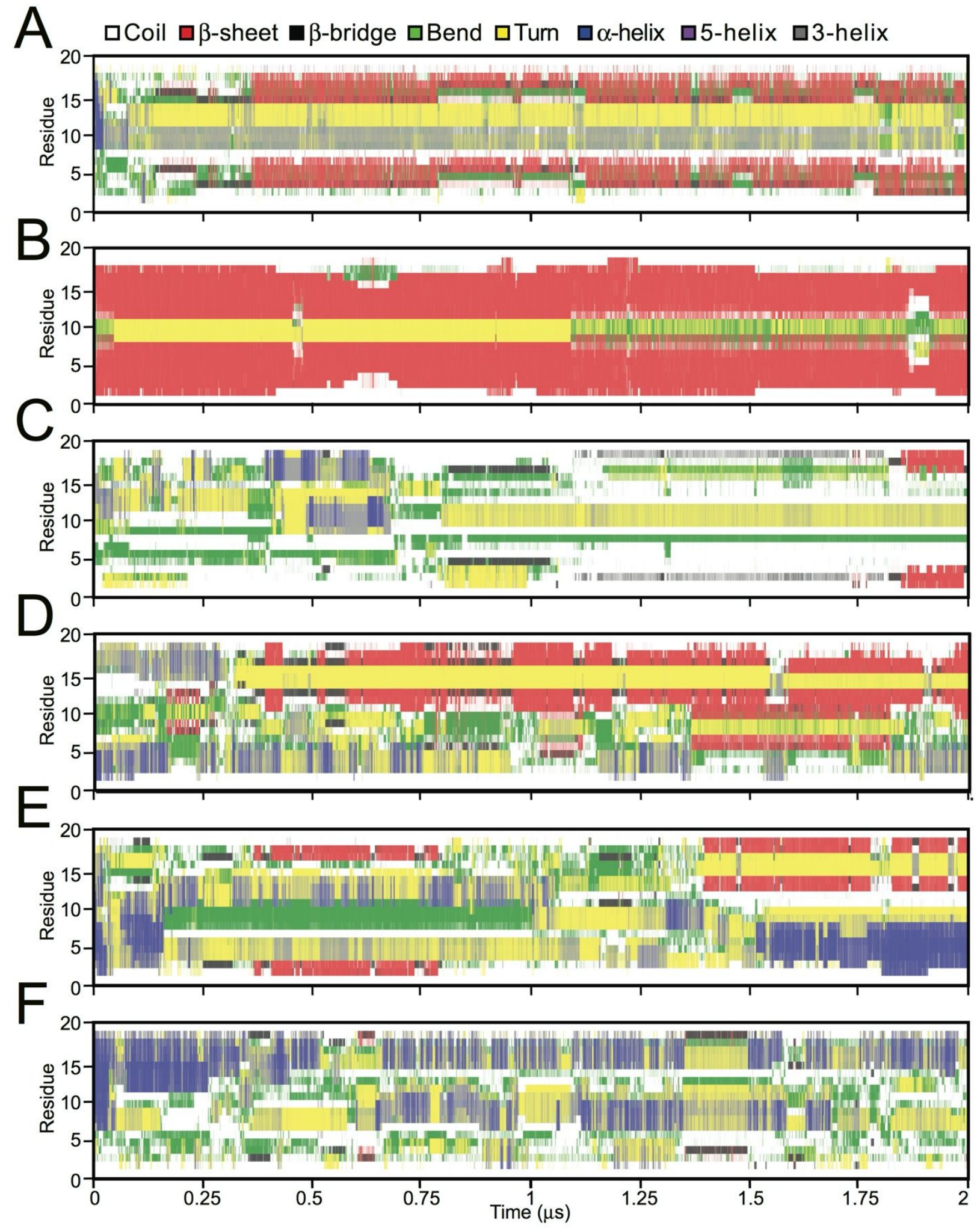




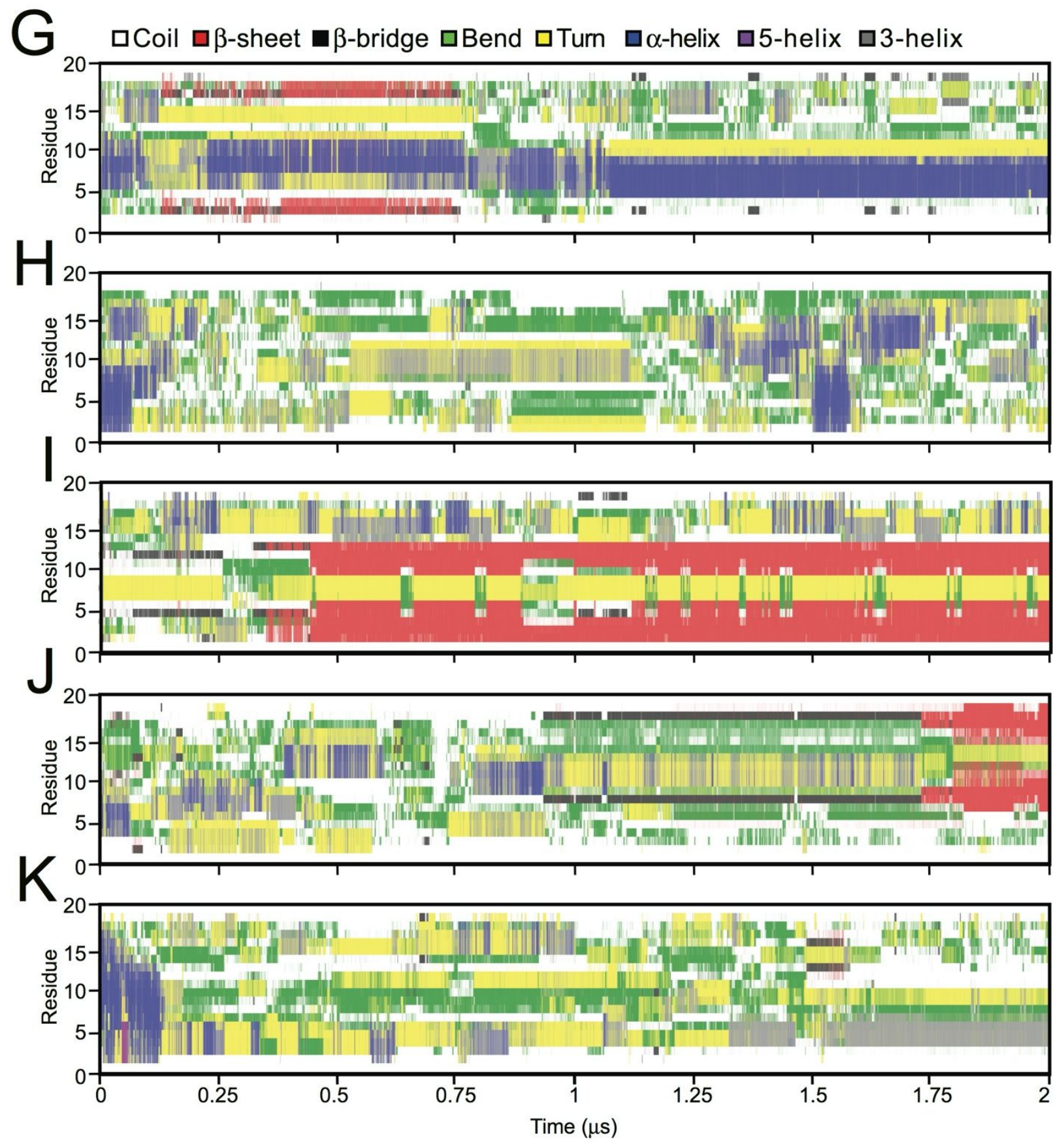




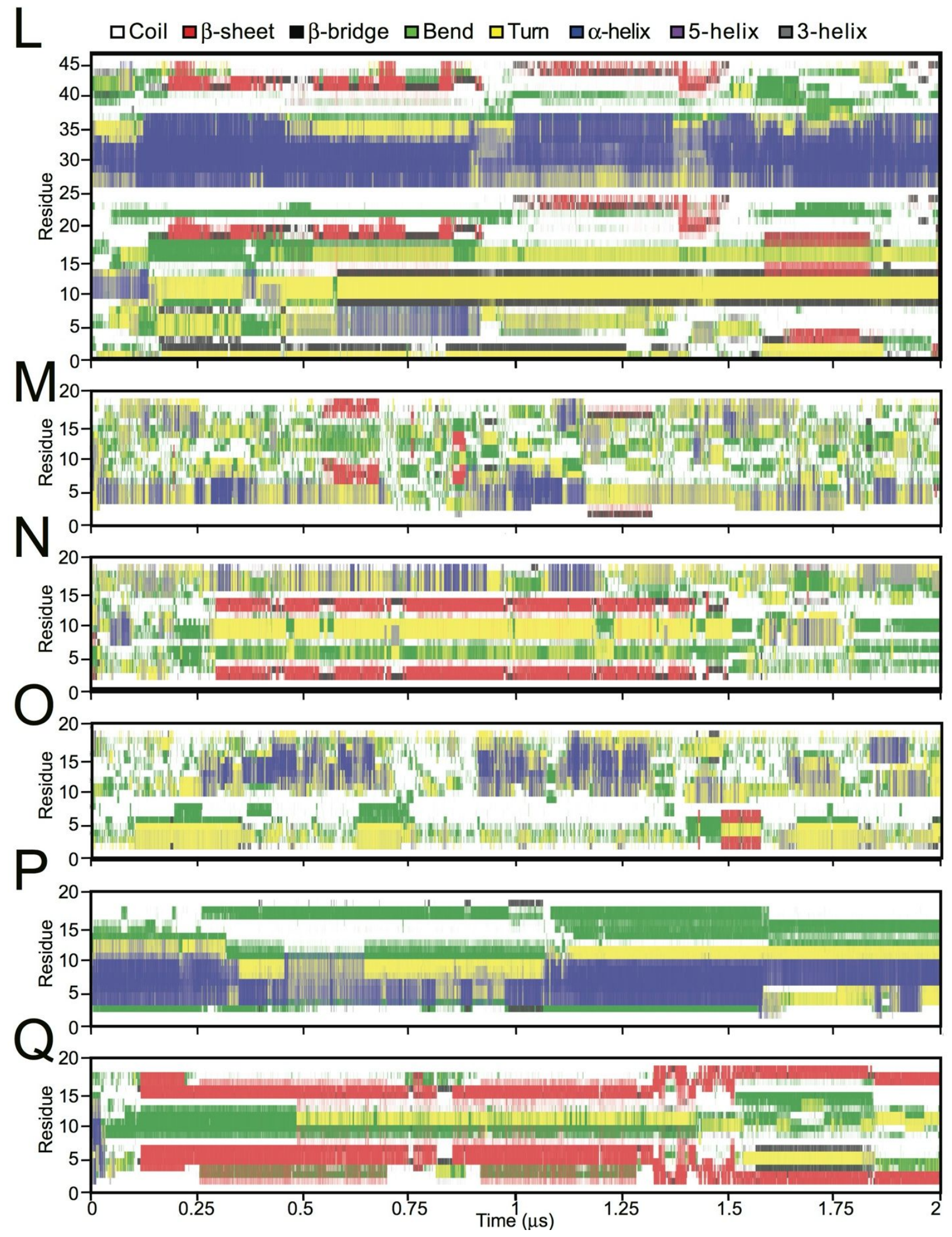


Supplemental Figure 4. Peptide secondary structure analysis during the $\mathbf{2} \boldsymbol{\mu}$ s MD simulation. Peptides A22 (A), A40 (B), A46 (C), A59 (D), A64 (E), A84 (F), A99 (G), A116 (H), A127 (I), A132 (J), A135 (K), A147 (L), A155 (M), A159 (N), A160 (O), A176 (P), and TRX-GST (Q) secondary structure analysis. Color output from DSSP: coil (white), $\beta$-sheet (red), $\beta$-bridge (black), bend (green), turn (yellow), $\alpha$-helix (blue), 5-helix (purple), and $3^{10}$-helix (grey). 
Supplemental Figure 5.

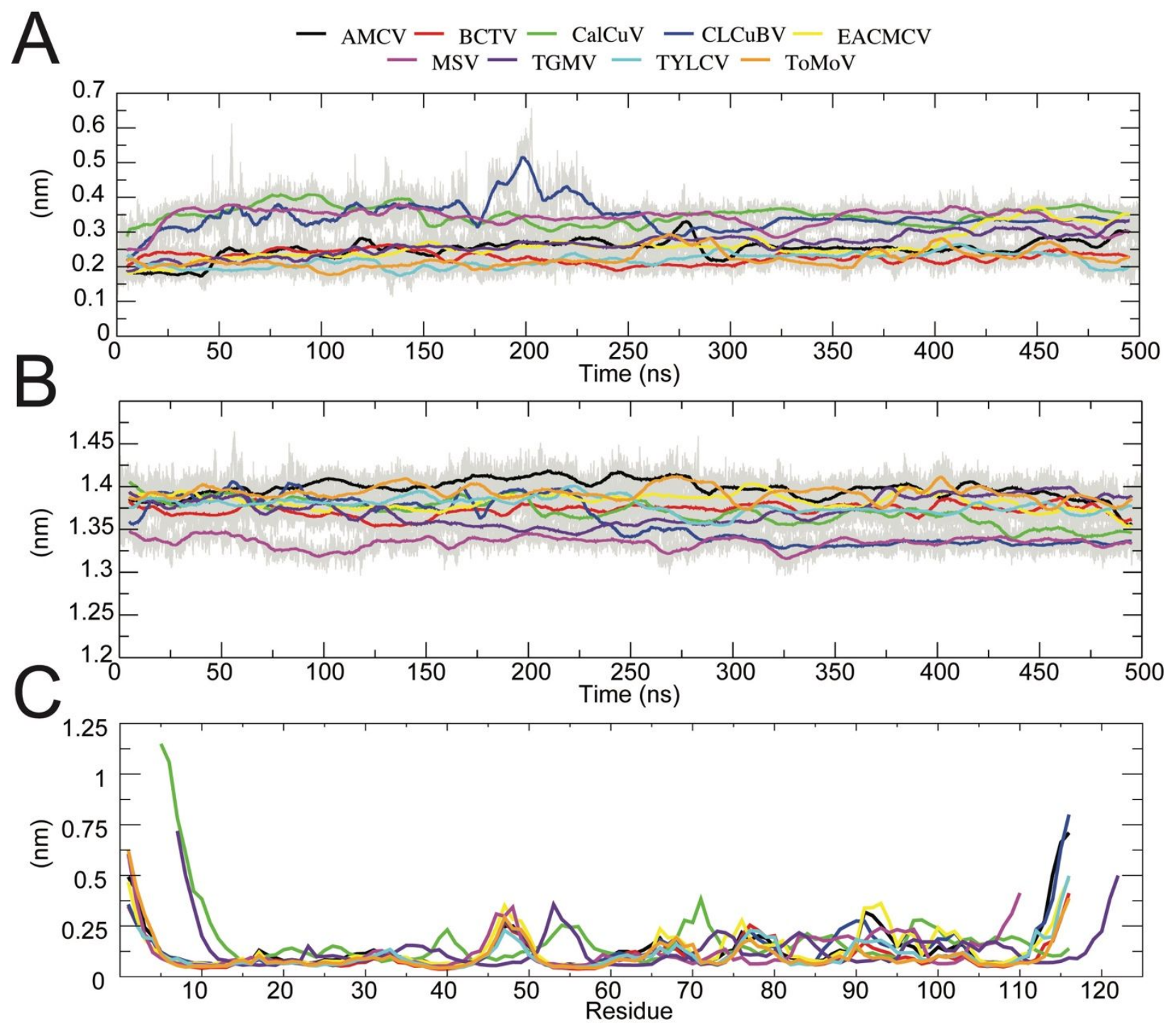

Supplemental Figure 5. N-term Rep model analysis of the 500ns protein MD simulation. (A) $\mathrm{C} \alpha-\mathrm{RMSD}$, (B) the radius of gyration (Rg), and (C) the C $\alpha$-RMSF per residue values over the length of the simulation. The lines are panels A-C are colored according to: AMCV (black), BCTV (red), CalCuV (green), CLCBuV (blue), EACMV (yellow), MSV (magenta), TGMV (purple), TYLCV (cyan), and ToMoV (orange). Raw data is shown in grey lines while colored lines are the running averages. 
Supplemental Figure 6.

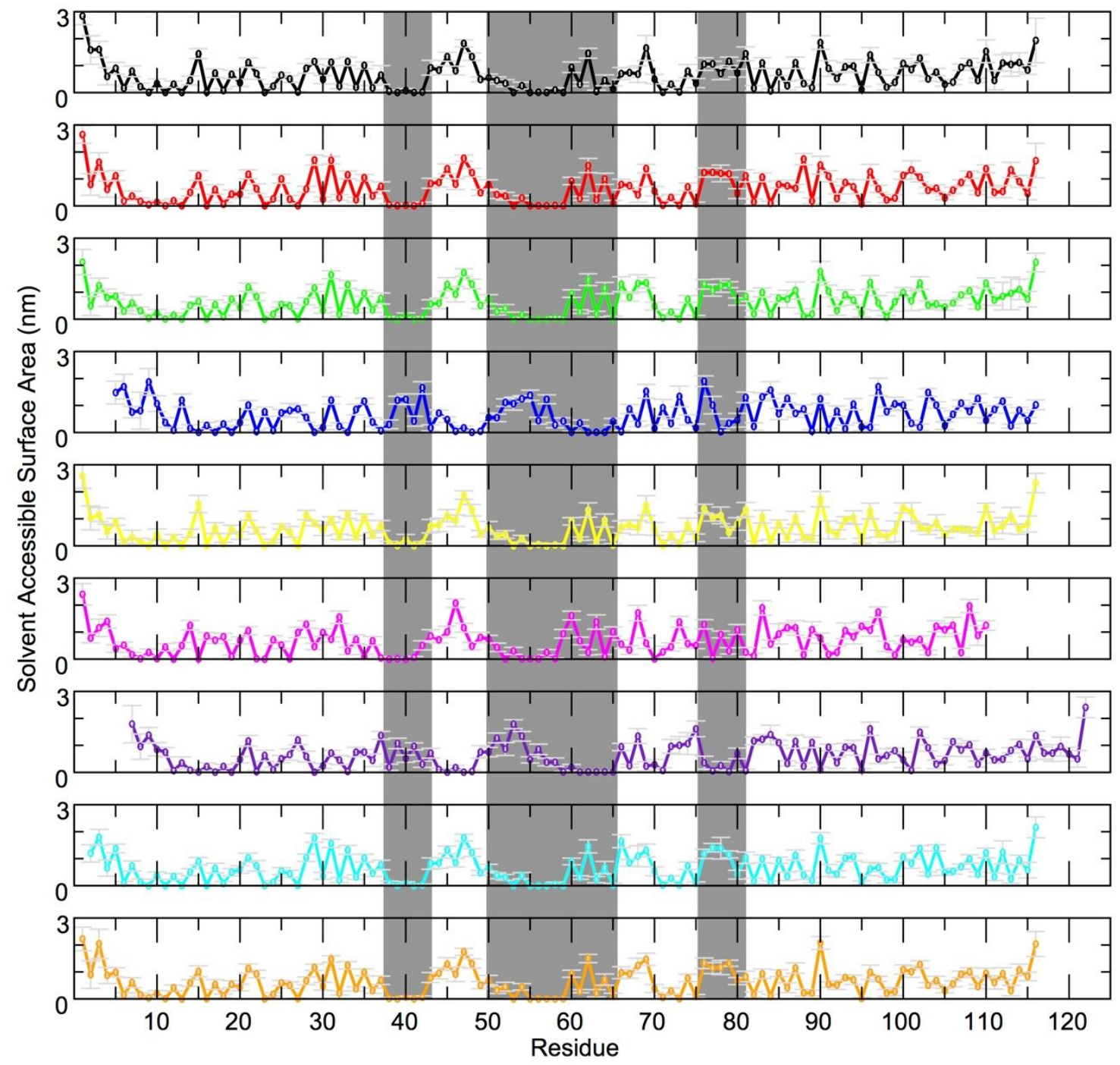

Supplemental Figure 6. Solvent accessibility surface area average and standard deviation as calculated through GROMACS over the length of the $2 \mu$ s MD simulation per residue in $\mathbf{N}$ term Rep models. From top to bottom: AMCV (black), BCTV (red), CLCBuV (green), $\mathrm{CalCuV}$ (blue), EACMV (yellow), MSV (magenta), TGMV (purple), TYLCV (cyan), and ToMoV (orange). Standard deviation is shown in light grey. Areas of notable difference of $\mathrm{CalCuV}$ and TGMV in comparison with the rest of the N-term Rep models are highlighted in grey. 


\section{Supplemental Figure 7.}

A

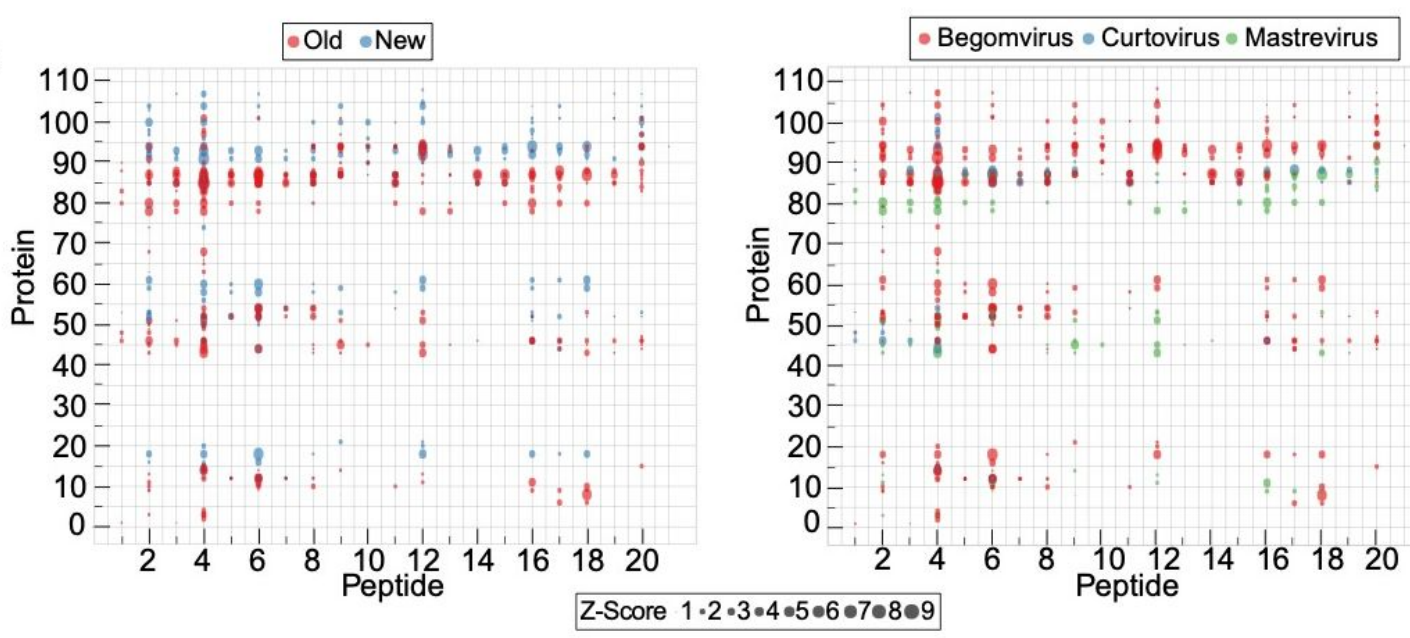

B

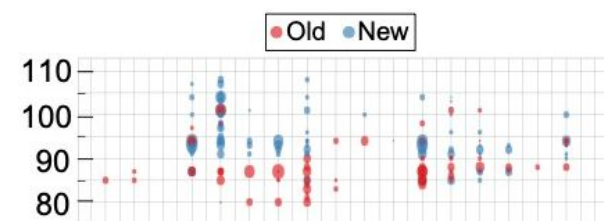

70
동 60
으 50

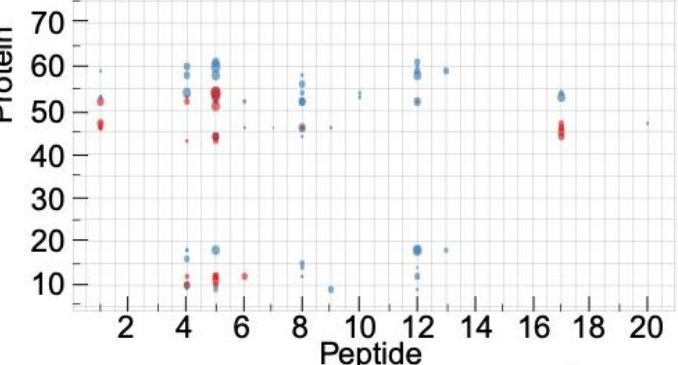

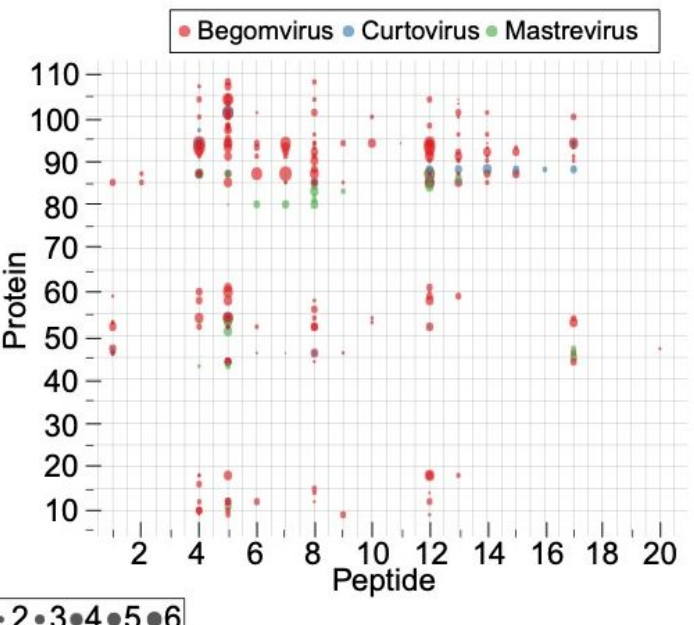

Z-Score $1 \cdot 2 \cdot 3 \cdot 4 \cdot 5 \cdot 6$

Supplemental Figure 7. 3D bubble chart protein and peptide Z-score interaction pairs. Each bubble represents an interaction between A22 (top) or A64 (bottom) with the protein of interest. The larger the bubble the bigger the Z-score and significance of the interaction. Sequences were aligned according to the alignment in Supplemental Figure S1. Legends for each chart are provided for clarity. 


\section{Supplemental Tables}

\begin{tabular}{|c|c|}
\hline \multicolumn{2}{|c|}{ Supplemental Table 1. Active residues during molecular docking } \\
\hline Protein/Peptide & Active residues \\
\hline ACMV & $\begin{array}{l}\text { 1,2,3,5,15,17,19,21,25,28,29,31,33,35,44,45,46,47,48,49,60,61,62,66,67,69,74,76,77,78,79,80,81,83,85,87, } \\
90,91,93,94,96,97,100,102,103,104,107,108,109,110,112,113,114,115,116\end{array}$ \\
\hline BCTV & $\begin{array}{l}1,3,5,15,17,21,25,29,31,33,35,44,45,47,48,49,60,62,64,66,67,69,76,77,78,79,81,83,85,86,88,90,91,93,96,97 \\
100,101,102,103,104,107,109,110,113,114,116\end{array}$ \\
\hline $\mathrm{CalCuV}$ & $\begin{array}{l}5,6,8,9,13,21,23,25,27,31,35,39,40,42,44,52,54,55,56,57,67,68,69,71,73,76,79,80,83,84,86,87,90,92,94,97 \\
98,99,100,103,104,107,109,112,115,116\end{array}$ \\
\hline CLCuBV & $\begin{array}{l}5,6,8,9,13,21,23,25,27,31,35,39,40,42,44,52,54,55,56,57,67,68,69,71,73,76,79,80,83,84,86,87,90,92,94,97 \\
98,99,100,103,104,107,109,112,115,116\end{array}$ \\
\hline EACMV & $\begin{array}{l}1,3,5,15,17,21,25,28,29,31,33,35,45,47,48,49,60,62,64,66,67,69,76,77,80,81,83,85,87,90,91,93,94,96,100 \\
101,102,103,107,109,110,113,115,116\end{array}$ \\
\hline MSV & $\begin{array}{l}1,2,3,4,14,16,18,21,28,30,31,32,34,43,44,45,46,47,48,49,59,60,61,63,65,68,73,74,76,78,80,83,84,85,86,87,90 \\
93,95,96,97,104,105,106,108,110\end{array}$ \\
\hline TGMV & $\begin{array}{l}7,9,11,21,23,25,27,31,34,35,37,39,41,51,52,53,54,55,56,66,68,72,73,74,75,82,83,84,85,87,89,91,93,94,96, \\
97,98,99,100,103,106,108,109,110,113,115,116,118,121,122\end{array}$ \\
\hline ToMoV & $\begin{array}{l}1,3,4,5,14,15,17,19,21,22,25,29,31,33,35,44,45,46,47,48,49,60,62,66,67,68,69,74,76,77,78,79,80,81,83,85,87 \\
, 90,91,93,94,96,97,100,101,102,103,107,109,110,112,114,115,116\end{array}$ \\
\hline TYLCV & $\begin{array}{l}2,3,5,15,17,21,25,28,29,31,33,35,44,45,47,48,49,60,62,66,67,68,69,74,76,77,78,79,81,83,85,87,90,91,93,94 \\
97,100,102,103,104,107,108,109,110,112,114,116\end{array}$ \\
\hline A22 & $1,2,3,4,5,6,8,9,10,12,13,16,17,18,19,20$ \\
\hline A40 & $1,2,3,5,7,8,9,10,11,13,14,15,16,17,19,20$ \\
\hline A46 & $1,2,3,5,6,7,9,10,12,13,14,16,17,20$ \\
\hline A59 & $1,2,3,4,6,8,9,11,12,13,14,15,16,17,19,20$ \\
\hline A64 & $1,5,6,8,9,10,13,14,17,19$ \\
\hline A84 & $1,2,3,4,5,6,7,8,9,11,12,13,15,16,17,18,19,20$ \\
\hline A99 & $1,2,3,6,7,10,11,12,14,15,18,20$ \\
\hline A116 & $1,2,3,4,5,6,7,9,10,12,14,15,16,17,18,19,20$ \\
\hline A127 & $1,2,4,6,7,8,9,11,15,16,17,18,19,20$ \\
\hline A132 & $2,3,4,6,8,9,11,12,13,14,15,16,17,18,19,20$ \\
\hline
\end{tabular}




\begin{tabular}{|r|l|}
\hline A135 & $1,4,8,9,10,11,12,15,16,17,18,19,20$ \\
\hline A147 & $2,4,9,10,11,19,20,22,24,26,28,29,30,33,36,37,40,42,44,45,46$ \\
\hline A155 & $1,4,5,6,8,9,10,11,12,13,14,15,16,17,18,19,20$ \\
\hline A159 & $1,2,6,8,9,10,11,12,15,16,17,19$ \\
\hline A160 & $2,3,4,5,6,7,8,9,10,11,12,13,14,15,16,17,18,19,20$ \\
\hline TRX-GST & $1,2,5,9,10,12,14,15,16,17,18,19,20$ \\
\hline
\end{tabular}




\begin{tabular}{|c|c|c|c|c|c|c|c|c|c|c|}
\hline \multicolumn{11}{|c|}{ Supplemental Table 2. Active residues during molecular dockinga } \\
\hline \multirow[b]{2}{*}{ Aptamer } & \multirow[b]{2}{*}{$\begin{array}{c}\text { TGMV- } \\
\text { Replication } \\
\% \text { (aprox) }\end{array}$} & \multicolumn{9}{|c|}{ Binds to Rep } \\
\hline & & TGMV & ToMoV & $\mathrm{CaLCuV}$ & $\mathrm{CLCuV}$ & EACMV & ACMV & TYLCV & BCTV & MSV \\
\hline A5 & 30 & & & & & & & & & \\
\hline A6 & 25 & & & & & & & & & \\
\hline $\mathrm{A} 22^{\mathrm{bc}}$ & 13 & Y & Y & Y & Y & Y & Y & Y & Y & Y \\
\hline A25 & 20 & & & & & & & & & \\
\hline A 27 & 60 & & & & & & & & & \\
\hline $\mathrm{A} 40^{\mathrm{b}}$ & 35 & $\mathrm{Y}$ & $Y$ & $Y$ & Y & $\mathrm{Y}$ & $\mathrm{N}$ & $\mathrm{Y}$ & $\mathrm{Y}$ & $\mathrm{Y}$ \\
\hline $\mathrm{A} 46^{\mathrm{b}}$ & 30 & $Y$ & $\mathrm{Y}$ & $Y$ & Y & $Y$ & $Y$ & $Y$ & $Y$ & $\mathrm{Y}$ \\
\hline A53 & 75 & & & & & & & & & \\
\hline $\mathrm{A} 59^{\mathrm{b}}$ & 49 & Y & Y & $\mathrm{Y}$ & $Y$ & Y & Y & Y & Y & Y \\
\hline A63 & 75 & & & & & & & & & \\
\hline $\mathrm{A} 64^{\mathrm{bc}}$ & 23 & $Y$ & Y & Y & $\mathrm{N}$ & $Y$ & $Y$ & Y & Y & $\mathrm{Y}$ \\
\hline A67 & 60 & & & & & & & & & \\
\hline A71 & 35 & & & & & & & & & \\
\hline $\mathrm{A} 84^{\mathrm{b}}$ & 20 & $\mathrm{Y}$ & $\mathrm{Y}$ & $Y$ & Y & $\mathrm{Y}$ & $Y$ & Y & Y & $\mathrm{Y}$ \\
\hline A94 & 35 & & & & & & & & & \\
\hline A99b & 20 & $\mathrm{Y} / \mathrm{N}$ & Y & $\mathrm{Y}$ & $\mathrm{N}$ & $\mathrm{N}$ & Y & Y & $\mathrm{N}$ & Y \\
\hline A101 & 52 & & & & & & & & & \\
\hline $\mathrm{A} 116^{\mathrm{b}}$ & 15 & Y & Y & Y & $\mathrm{N}$ & Y & Y & Y & Y & $\mathrm{Y}$ \\
\hline A127 & & Y & Y & Y & Y & Y & $\mathrm{N}$ & Y & Y & Y \\
\hline A130 & 75 & & & & & & & & & \\
\hline A131 & 10 & & & & & & & & & \\
\hline $\mathrm{A} 132^{\mathrm{b}}$ & 45 & Y & Y & $\mathrm{Y}$ & Y & Y & $\mathrm{N}$ & Y & Y & Y \\
\hline $\mathrm{A} 135^{\mathrm{b}}$ & & Y & Y & Y & $Y$ & Y & $\mathrm{N}$ & Y & Y & Y \\
\hline A137 & 59 & & & & & & & & & \\
\hline A140 & 40 & & & & & & & & & \\
\hline A146 & 45 & & & & & & & & & \\
\hline
\end{tabular}




\begin{tabular}{|c|c|c|c|c|c|c|c|c|c|c|}
\hline $\mathrm{A} 147^{\mathrm{b}}$ & 12 & Y & $\mathrm{Y}$ & Y & $\mathrm{Y}$ & $\mathrm{Y}$ & $\mathrm{Y}$ & Y & $\mathrm{Y}$ & Y \\
\hline A153 & 75 & & & & & & & & & \\
\hline $\mathrm{A} 155^{\mathrm{b}}$ & 28 & Y & $\mathrm{N}$ & Y & $\mathrm{Y}$ & $\mathrm{Y}$ & $\mathrm{Y}$ & Y & $\mathrm{Y}$ & Y \\
\hline $\mathrm{A} 159^{\mathrm{b}}$ & 28 & Y & $\mathrm{N}$ & Y & Y & $\mathrm{Y}$ & $\mathrm{Y}$ & Y & $\mathrm{Y}$ & Y \\
\hline $\mathrm{A} 160^{\mathrm{b}}$ & 14 & Y & $\mathrm{N}$ & Y & Y & $\mathrm{Y}$ & $\mathrm{Y}$ & Y & $\mathrm{Y}$ & Y \\
\hline A168 & 53 & & & & & & & & & \\
\hline A169 & 40 & & & & & & & & & \\
\hline A173 & 30 & & & & & & & & & \\
\hline A174 & 33 & & & & & & & & & \\
\hline $\mathrm{A} 176^{\mathrm{b}}$ & 58 & $\mathrm{Y}$ & $\mathrm{N}$ & Y & $\mathrm{Y}$ & $\mathrm{Y}$ & $\mathrm{Y}$ & $\mathrm{Y}$ & $\mathrm{Y}$ & $\mathrm{Y}$ \\
\hline A177 & 53 & & & & & & & & & \\
\hline
\end{tabular}

\section{aReferences:}

Lopez-Ochoa L, Ramirez-Prado J, Hanley-Bowdoin L. Peptide aptamers that bind to a geminivirus replication protein interfere with viral replication in plant cells. J Virol. 2006 Jun;80(12):5841-53. doi: 10.1128/JVI.02698-05. PMID: 16731923; PMCID: PMC1472579.

Reyes MI, Nash TE, Dallas MM, Ascencio-Ibáñez JT, Hanley-Bowdoin L. Peptide aptamers that bind to geminivirus replication proteins confer a resistance phenotype to tomato yellow leaf curl virus and tomato mottle virus infection in tomato. J Virol. 2013 Sep;87(17):9691-706. doi: 10.1128/JVI.01095-13. Epub 2013 Jul 3. PMID: 23824791; PMCID: PMC3754110.

bUsed in this work

'Tested in transgenic plants 
Supplemental Table 3. Z-Scores higher than 3 for peptide:protein pairs

\begin{tabular}{|c|c|c|c|c|c|c|c|}
\hline \multirow[t]{6}{*}{ Peptide } & Organism & Peptide & Protein & Peptide & Organism & Peptide & Protein \\
\hline & \multirow{5}{*}{ ACMV } & 3 & 85 & \multirow{36}{*}{ A64 } & \multirow{5}{*}{ ACMV } & & \\
\hline & & 4 & $68,83,85-87$ & & & & \\
\hline & & 5 & 85 & & & & \\
\hline & & 6 & $12,52,85$ & & & & \\
\hline & & 11,14 & 87 & & & & \\
\hline \multirow{31}{*}{ A22 } & \multirow{9}{*}{ BCTV } & 2 & 46 & & \multirow{9}{*}{ BCTV } & 4 & 87,94 \\
\hline & & 3 & 88 & & & 5 & 54,101 \\
\hline & & 4 & $\begin{array}{c}44,87,88 \\
94,101\end{array}$ & & & 12 & 85 \\
\hline & & 5 & 87 & & & 14 & 88 \\
\hline & & 6 & $85-88$ & & & & \\
\hline & & 7 & 85 & & & & \\
\hline & & 8 & $85-87$ & & & & \\
\hline & & 11,15 & 85 & & & & \\
\hline & & 17,18 & 88 & & & & \\
\hline & \multirow{5}{*}{ CalCuV } & 2 & $61,92,94$ & & \multirow{5}{*}{ CalCuV } & 4 & 92,94 \\
\hline & & 12 & $\begin{array}{c}18,61,92,94, \\
104\end{array}$ & & & 5 & $\begin{array}{c}61,92,94, \\
104\end{array}$ \\
\hline & & 16 & $18,92,94$ & & & 7 & 94 \\
\hline & & 17 & 94 & & & 12 & $18,92,94$ \\
\hline & & 18 & $18,59,61,94$ & & & 14 & 92 \\
\hline & \multirow{4}{*}{ CLCuBV } & 2 & 87,91 & & \multirow{4}{*}{ CLCuBV } & & \\
\hline & & 9 & 94 & & & & \\
\hline & & 12 & 93,94 & & & & \\
\hline & & 18 & 8 & & & & \\
\hline & \multirow{7}{*}{ EACMV } & 3 & 85,87 & & \multirow{7}{*}{ EACMV } & 5 & 85 \\
\hline & & 4 & $85-87$ & & & 6,7 & 87 \\
\hline & & 5 & 87 & & & 8 & 87,90 \\
\hline & & 6 & $12,54,85,87$ & & & 10 & 94 \\
\hline & & 8 & 52,54 & & & & \\
\hline & & 12 & 94 & & & & \\
\hline & & 14,15 & 87 & & & & \\
\hline & \multirow{6}{*}{ MSV } & 2 & $51,78,80$ & & \multirow{6}{*}{ MSV } & 4 & 93 \\
\hline & & 3 & 80 & & & 5 & $\begin{array}{c}18,58,60 \\
91,93\end{array}$ \\
\hline & & 4 & $\begin{array}{c}43,45,51,78 \\
80\end{array}$ & & & 7 & 93 \\
\hline & & 6 & 11,87 & & & 12 & $58,91,93$ \\
\hline & & 9 & 45,87 & & & 17 & 53 \\
\hline & & 12 & $43,51,78$ & & & & \\
\hline
\end{tabular}




\begin{tabular}{|c|c|c|c|c|c|}
\hline & 16 & $11,80,87$ & & & \\
\hline & $17-19$ & 87 & & & \\
\hline \multirow{5}{*}{ TGMV } & 2 & 100 & \multirow{5}{*}{ TGMV } & 4 & 93 \\
\hline & 3 & 93 & & 5 & $\begin{array}{c}18,58,60 \\
91,93\end{array}$ \\
\hline & 4 & $\begin{array}{c}8,58,60,91 \\
93,100,104\end{array}$ & & 7 & 93 \\
\hline & 6 & $\begin{array}{c}16,18,58,60 \\
91,93\end{array}$ & & 12 & $58,91,93$ \\
\hline & $14-15$ & 93 & & 17 & 53 \\
\hline \multirow{6}{*}{ ToMoV } & 4 & $14,46,52,85$ & \multirow{6}{*}{ ToMoV } & 4 & 54 \\
\hline & 7 & $12,44,52,54$ & & 5 & 97,101 \\
\hline & 8,9 & 87 & & 8 & 46 \\
\hline & 16 & 46 & & 12,13 & 85 \\
\hline & 20 & 94 & & 15 & 87 \\
\hline & & & & 17 & 94 \\
\hline \multirow{3}{*}{ TYLCV } & 4 & 14,85 & \multirow{3}{*}{ TYCLV } & 5 & 54 \\
\hline & 6 & $12,44,52,54$ & & 8 & 85 \\
\hline & 20 & 94 & & 12 & 85,87 \\
\hline
\end{tabular}

